\title{
DIETARY HABITS IN A GREEK SAMPLE OF MEN AND WOMEN: THE ATTICA STUDY
}

\author{
Fotini Arvaniti ${ }^{1}$, Demosthenes B. Panagiotakos ${ }^{1}$, Christos Pitsavos ${ }^{2}$, Antonis Zampelas ${ }^{1}$, Christodoulos Stefanadis ${ }^{2}$ \\ ${ }^{1}$ Department of Nutrition - Dietetics, Harokopio University, Athens, Greece \\ ${ }^{2}$ First Cardiology Clinic, School of Medicine, University of Athens, Athens, Greece
}

\section{SUMMARY}

Objective: Although there are several dietary guidelines accepted at the international and national level, there are indications that dietary habits in Greece have been changing, moving away from the traditional Mediterranean diet pattern. The aim of this work was to evaluate the dietary habits of a randomly selected sample from the general adult population, in Greece.

Subjects: The "ATTICA" study is a prospective health and nutrition survey. For the purpose of this study, 3,042 adults, from whom 1514 were men (48\%) and 1,528 were women (52\%), were selected from the greater Athens area during 2001 and 2002. The assessment of the dietary habits was carried out using a validated food frequency questionnaire.

Results: Dietary intakes of red meat and sweets were higher $(p<0.005)$, and dietary intakes of fish, poultry, dairy products, vegetables, and cereals were lower $(p<0.05)$ than the ones recommended by the Hellenic Ministry of Health.

Conclusion: The results from the present study indicate a change in the dietary habits towards an unhealthier type of diet.

Key words: dietary habits, Greece, food groups, Mediterranean diet

Address for correspondence: D. B. Panagiotakos, 46 Paleon Polemiston St., 166 74, Attica, Greece. E-mail: d.b.panagiotakos@usa.net

\section{INTRODUCTION}

The dietary guidelines implemented in any country have as main aim the prevention of diseases through the consumption of a balanced diet, and the decrease in nutritional inadequacies. A major part of the nutritional policy is the development of national nutritional guidelines, adapted to the needs of each country, which could be easily understood by the public (1). The World Health Organization, as well as various other countries, including Greece, have already published dietary guidelines for their citizens. These guidelines share common characteristics, such as the increase in fruits, vegetables and cereals consumption, the decrease in total fat intake, and in particular the saturated fat intake, the substitution of red fatty meat with poultry and fish, the moderate consumption of low-fat dairy products, the decrease in sugar and salt intakes and the moderate consumption of alcohol. In Greece, dietary guidelines have been suggested by the Ministry of Health in 1999 (2), and are presented to the public in a form of a dietary pyramid, resembling the so called "Mediterranean Diet" model.

However, during the last 4 decades, there is a wide belief that significant changes have been made in the dietary habits in Greece, and have diversified the habits of modern Greeks from those of their ancestors. In addition, several researchers have associated these changes with an increase in nutrition related diseases, such as cardiovascular diseases, hypertension, diabetes mellitus, obesity, and some types of cancer $(3,4)$. However, there is only a limited number of surveys looking at the dietary habits of the Greek population. In particular, the 7 Countries Study has been assessing the changes in the dietary habits in populations from 7 countries since 1960, including two cohorts of about 1,300 men from two
Greek islands (Corfu and Crete) (3). Another study assessed the dietary habits of 387 Cretans in 1980 (4). Furthermore there are data from household budget surveys (5) and from a pan-European study, the EPIC, which enrolle about 22,000 adult men and women from various Greek regions (6).

Therefore, the aim of the present work was to evaluate the dietary intake of a randomly selected and representative sample of men and women from the Athens greater area and compare it with the recent dietary guidelines for the Greek population.

\section{MATERIALS AND METHODS}

\section{Study Population}

The "ATTICA" epidemiological study (7) was carried out in the province of Attica (including 78\% urban and 22\% rural areas), where Athens is the metropolis. From May 2001 to December 2002, a total number of 4,056 inhabitants were randomly selected to enrol in the study, 3,042 of whom consented to participate ( $75 \%$ participation rate). All participants were interviewed and examined by specialists (cardiologists, general practitioners, dieticians and qualified nurses) and further data were collected by means of a questionnaire and of laboratory tests. Five percent of men and three percent of women were excluded from the present analysis because they reported a history of cardiovascular or some other atherosclerotic disease and chronic viral infections. Moreover, the rest of the participants had not had a cold or the flu, acute respiratory infection, dental problems or any type of surgery the week before the data collection. People living in institutions were also excluded from the sampling. Power analysis showed that the number of the participants is adequate to evaluate two-sided standardised differences between subgroups of the study and that the investigated parameters greater than 0.5 achieved statistical power greater than 0.90 at $5 \%$ probability level (P-value).

The study was approved by the Medical Research Ethics Committee of the First Cardiology Clinic, School of Medicine, University of Athens and was carried out in accordance with the Declaration of Helsinki (1989) of the World Medical Association. 


\section{Dietary Ascertainment}

Dietary assessment was based on a validated semi-quantitative food frequency questionnaire (FFQ) (8). ${ }^{*}$ The questionnaire assessed usual dietary intake of 156 foods and beverages commonly consumed in Greece, with 7 non-overlapping response categories. Photographs assisted the responders to define the portion sizes in several foods which were included in the questionnaire. In particular, consumption of non-refined cereals and products (like whole grain bread, pasta, rice, etc), vegetables, legumes, fruit, dairy products (like cheese, yoghurt, milk), nuts, potatoes, eggs, sweets, fish, poultry, red meat and meat products, use of olive oil in cooking, as well as coffee and alcohol drinking were measured as an average per week during the past year. Frequency of consumption was quantified in terms of the number of times a month a food was consumed in small, medium or large portion sizes. Alcohol consumption was recorded as daily ethanol intake of $100 \mathrm{ml}$ wineglasses adjusted for $12 \%$ ethanol concentration.

According to the Mediterranean dietary pattern provided by the Supreme Scientific Health Council, Ministry of Health and Welfare of Greece (2) it is suggested: (a) daily consumption of: non refined cereals and products (whole grain bread, pasta, brown rice, etc), fruit (servings/day), vegetables (servings/day), olive oil (as the main lipid added) and dairy products ( 2 servings/day), (b) weekly consumption of: fish, poultry, potatoes, olives, pulses, and nuts, and more rarely eggs and sweets (3 servings/week) and monthly consumption of: red meat and meat products (servings/month). It is also suggested moderate consumption of alcoholic beverages (12-24 g of ethanol/ day), especially wine, and moderate consumption of fat. In accordance with that dietary pattern and the reported monthly frequency consumption of these food groups considered, we calculated a diet score (9) that assessed adherence to the Mediterranean diet for each participant. In particular, for the consumption of items presumed to be "close" to this pattern (i.e. those suggested on a daily basis or more than 4 servings per week) we assigned score 0 when someone reported no consumption, score 1 when they reported consumption of 1 to 4 times per month, score 2 for 5 to 8 times, score 3 for 9 to
12 times per month, score 4 for 13 to 18 times per month and score 5 for more than 18 times per month. On the other hand, for the consumption of food items presumed to be "away" from this diet guidelines (like meat and meat products) we assigned the scores on a reverse scale (i.e. 0 when a participant reported almost daily consumption and 5 for rare or no consumption). Especially for alcohol we assigned score 5 for consumption of less than 3 wineglasses per day, score 0 for consumption of more than 7 wineglasses per day and scores 1 to 4 for consumption of 3, 4-5, 6 and 7 wineglasses per day. Higher values (range $0-55$ ) of this special diet score mentioned above indicate greater adherence to the Mediterranean diet.

Additionally, based on food composition tables (10) and the retrieved information we calculated daily energy intake (in kcal), total, monounsaturated, saturated, polyunsaturated lipids (in g per day), the percentage of energy from saturated lipids and the ratio of monounsaturated - to - saturated lipids.

\section{Statistical analysis}

Continuous variables are presented as mean values \pm standard deviation, while categorical variables are presented as absolute and relative frequencies. Comparisons between participants' consumption and dietary guidelines were performed by the use of Student's t-test. All reported p-values were based on two-sided tests and compared to a significance level of 5\%. SPSS 11.0.5 (SPSS Inc., Chicago, IL, USA) software was used for all the statistical calculations.

\section{RESULTS}

As we can see in Table 1, both men and women consumed significantly more $(p=0.03)$ red meat and less white lean meat $(p<0.05)$ than the recommended dietary intake. In particular, participants consumed about 3 portions of fish and 2 portions of poultry less than the recommended intake for the general population. In addition, further analysis showed that only $10 \%$ of the subjects had an acceptable red meat intake (1 serving per week) and less

Table 1. Food consumption in the study sample

\begin{tabular}{|c|c|c|c|}
\hline \multirow[t]{2}{*}{ Food } & \multirow[t]{2}{*}{ Dietary recommendations (2) } & $\begin{array}{c}\text { Men } \\
(n=1,514)\end{array}$ & $\begin{array}{c}\text { Women } \\
(n=1,528)\end{array}$ \\
\hline & & \multicolumn{2}{|c|}{ Servings / week } \\
\hline Red meat and products & 1 serving/week & $4.8 \pm 2.7^{\star}$ & $4.0 \pm 2.2^{*}$ \\
\hline Poultry & 4 servings/week & $1.4 \pm 0.1^{*}$ & $1.5 \pm 0.2^{*}$ \\
\hline Fish & 6 servings/week & $2.1 \pm 1.1^{*}$ & $2.0 \pm 1.2^{*}$ \\
\hline Eggs & 3-4 servings/week & $1.1 \pm 0.7^{\star}$ & $1.2 \pm 1^{*}$ \\
\hline Nuts & 1 serving/week & $1.3 \pm 2.0$ & $1.4 \pm 2.1$ \\
\hline Legumes & 3-4 servings/week & $5.4 \pm 3.0$ & $4.7 \pm 2.2$ \\
\hline Dairy products & 14 servings/week & $11.5 \pm 5^{\star}$ & $12.9 \pm 5^{*}$ \\
\hline Fruit & 21 servings/week & $26 \pm 3^{*}$ & $28 \pm 4^{*}$ \\
\hline Vegetables & 42 servings/week & $34 \pm 15^{*}$ & $35 \pm 13^{*}$ \\
\hline Potatoes & 21 servings/week & $13 \pm 4^{*}$ & $14 \pm 3^{*}$ \\
\hline Cereals & 56 servings/week & $52 \pm 18^{*}$ & $53 \pm 18^{*}$ \\
\hline Use of olive oil in cooking & Use on a daily basis & $88 \%$ & $87 \%$ \\
\hline Alcohol (g of ethanol / day) & 15-30 g/day & $12.5 \pm 4$ & $5.5 \pm 3$ \\
\hline Sweets & 3 servings/week & $4.9 \pm 4^{*}$ & $4.8 \pm 4^{*}$ \\
\hline Mediterranean diet score $(0-55)$ & & $25 \pm 3$ & $27 \pm 3$ \\
\hline
\end{tabular}

${ }^{*} p<0.05$ compared to the recommended dietary intakes

${ }^{* *}$ FFG has been kindly provided by the Laboratory of Epidemiology, School of Medicine, University of Athens. 
than $1 \%$ of the subjects met the recommended intake for fish (5-6 servings per week). It was also observed that men consumed more red meat than women $(\mathrm{p}<0.001)$. Dairy products, vegetable and cereals consumption was found to be lower than the recommended dietary intakes $(p<0.001)$ in both genders. In addition, only $30 \%$ of the participants met the recommendations for dairy products (14 portions per week) and only $35 \%$ for cereals ( 56 portions per week). Women consumed more dairy products than men ( $<<0.001)$. Fruit and legume intake was found to be within the recommended range in both genders. Similar results were observed regarding alcoholic beverages intake. Finally an increased consumption of sweets was observed in both sexes $(\mathrm{p}<0.005) .75 \%$ of the subjects were consuming more than the recommended intake ( 3 portions per week). Moreover, $88 \%$ of men and $87 \%$ of women were using olive oil as the main added fat in their diet.

The dietary score which indicates adherence to the Mediterranean diet was found to be relatively low (about 26/55) in men and women. However, women had a higher score than men (Table 1). Moreover, an inverse association between diet score and age was observed in both genders ( $r$ for men $=-0.34, p<0.001$, and $r$ for women $=-0.48, \mathrm{p}<0.001)$.

Percentage of energy intake due to total lipids was $45 \%$ in men and $47 \%$ in women. Moreover, Table 2 illustrates total energy and other lipids intake in men and women. As we can see men had higher energy intake and lower monounsaturated: to saturated lipid ratio compared to women. On the other hand women had lower lipid intake (monounsaturated, saturated and polyunsaturated) compared to men.

\section{DISCUSSION}

The results of this work indicate that the population-based sample studied significantly deviated from the recommended dietary guidelines for Greek adults. In particular, in both genders a higher intake in red meat and a lower intake in white meat, including fish and poultry were observed. Additionally, a lower intake in

Table 2. Nutrient intake in a sample of 3,042 men and women from Greece

\begin{tabular}{|l|c|c|}
\hline & $\begin{array}{c}\text { Men } \\
(\mathbf{n}=1,514)\end{array}$ & $\begin{array}{c}\text { Women } \\
(\mathbf{n}=1,528)\end{array}$ \\
\hline Daily energy intake (in kcal) & $2,595 \pm 877$ & $2,132 \pm 658^{*}$ \\
\hline Protein (g/day) & $88 \pm 39$ & $76 \pm 37$ \\
\hline$\%$ of total energy & 14 & 14 \\
\hline Carbohydrate (g/day) & $222 \pm 102$ & $198 \pm 90$ \\
\hline$\%$ of total energy & 34 & 37 \\
\hline Total lipids (g/day) & $130 \pm 47$ & $112 \pm 60$ \\
\hline$\%$ of total energy & 45 & 47 \\
\hline Monounsaturated lipids (g/day) & $70 \pm 40$ & $59 \pm 33^{\star}$ \\
\hline$\%$ of total energy & 24 & 25 \\
\hline Saturated lipids (g/day) & $37 \pm 20$ & $32 \pm 20^{\star}$ \\
\hline$\%$ of total energy & 13 & 14 \\
\hline Polyunsaturated lipids (g/day) & $18 \pm 10$ & $15 \pm 8^{\star}$ \\
\hline$\%$ of total energy & 6 & 6 \\
\hline $\begin{array}{l}\text { Monounsaturated - to - saturated } \\
\text { fat ratio }\end{array}$ & $1.95 \pm 1.1$ & $1.89 \pm 1.7^{\star}$ \\
\hline
\end{tabular}

* $p<0.05$ for the comparisons between genders dairy products, vegetables and whole grain food (cereals) items than the one recommended was found. Moreover, an increased intake of sweets was also observed. The consumption of fruits was acceptable in both sexes and the majority of the subjects were using olive oil as the main added fat.

The main differences in the consumption were observed in foods that are associated, either positively or negatively, with the development of chronic diseases, such as cardiovascular diseases, diabetes and cancer. The high consumption of red meat and products confirms the indication that Greek people tend to consume an unhealthier type of diet than Greeks in the mid 1950's and this is in accordance with the results of other studies $(4,6,11)$. These results are in accordance with the results from the Greek cohort of the EPIC Study which also showed that consumption of red meat is higher than the recommended one (6). However, there was no distinction between red and white meat consumption.

Eggs consumption was found to be low as regards the dietary recommendations (Table 1). Moreover, the results from our study are in contrast with the results observed by the 7 Countries Study in the early 1960s where a consumption of 1 egg per day was observed (3). Additionally, in the EPIC Study consumption of 1 egg every other day also was also observed (6).

Diary products consumption was lower than the recommended ones. This may lead to a decreased calcium intake by the people. The results from the present study are in accordance with those regarding the Greek cohorts from the 7 Countries Study observed 4 decades before (3) as well as from the recent EPIC Study (6), too.

Vegetable consumption is much lower than the quantity recommended for the Greek population. However, recommendations vary among countries and it should be noted that according to the European (12) and American (13) recommendations, vegetable consumption in Greece is at an acceptable level. The results of the present study are also in accordance with the results of the Greek cohorts of the EPIC Study (6) and of the 7 Countries Study (3). It is noteworthy that vegetable intake of the Cretan cohort of the 7 Countries Study in the 1960's was only 190 g per day which is considered low. However, fruit consumption was high (464 g per day), which has also been observed in our study.

Consumption of foods rich in carbohydrates was found to be low, which is in agreement with the results of the EPIC Study. On the contrary, legume consumption was in an acceptable range in our work, whilst the results of the EPIC Study (3) showed much lower legume intake.

The vast majority of the participants $(90 \%)$ were using olive oil as the added fat in their diets, which is in accord with the results from the EPIC Study that also reported $46 \mathrm{~g}$ olive oil consumption per day for men and $39 \mathrm{~g}$ for women. In contrast, results from the 7 Countries Study in the early 1960s indicated a much higher consumption which reached the level of $95 \mathrm{~g}$ per day (3).

Sweet consumption was found to be high. In both men and women sweet consumption was higher than the recommended one (Table 1) and $80 \%$ of the participants consumed more sweets than it was recommended.

Afterwards we evaluated dietary habits of the participants in a holistic way through a special diet score. The relatively low mean level of the score, observed in this study, reflects the elevated consumption of red meat and its products and the low consumption of fish, poultry, vegetables, dairy and whole grain products. It has also to be mentioned that a higher score was observed in women 
than in men, mainly due to the lower red meat and the higher dairy products and fruit consumption. The dietary score was also found to be decreasing with age. This could be due to the increasing loss of interest, chewing difficulties, especially of fruits and vegetables, difficulties in the preparation of the meals and other. The financial problems that people face after the retirement could lead to the decrease in the consumption of foods that althouth considered healthy are for them expensive, such as fish. Our results are in contrast with the results of some studies which showed better dietary habits in the elderly compared to the adults $(6,14-17)$. However, in another big study it was observed that elderly people aged 70-90 years, had an average Mediterranean diet score 4 out of 8 also due to an increased consumption of red meat and a low consumption of fish and vegetables (17).

As regards total energy intake this seems to be little lower than the recommended one (i.e. $2,750 \mathrm{kcal} /$ day for men and 2,130 kcal/ day for women) $(1,2)$. Total fat intake was found to be high but almost half of it was in the form of monounsaturated fatty acids (Table 2). Saturated fat intake was also higher than the recommendations but polyunsaturated fat intake was acceptable. Since dairy products intake was found to be below the recommendations, it could be assumed that the main source of saturated fat was the meat and its products. On the other hand, the daily use of olive oil in the salad is a tradition which is still alive and it provides the population with the necessary monounsaturated fat in the diet. However, the low consumption of fish indicates that $\omega-3$ fatty acids intake may be unacceptably low and this may have an impact on the risk of several diseases including cardiovascular diseases. Carbohydrate intake is relatively low but far more than the lower limit of $100 \mathrm{~g}$ per day. However, since cereal and vegetable intakes are lower than the recommended ones there is a possibility that dietary fibre intake may be lower than $20-30$ $\mathrm{g}$ /day. Protein intake was within the recommended range (10-20\% as energy) for both men and women and the main contributors would be the meat and its products as well as the legumes. This finding is in agreement with the wide belief that there is no problem with protein intake in the westernized societies. Finally, sweet consumption was found to be high and this is a good source of fat and simple sugars. Unfortunately, there are no data on beverage intake.

\section{LIMITATION OF THE STUDY}

Although, the sample of the present study is large and randomly selected from the general population of the Athens greater area (that has about the $50 \%$ of the Greek population) we cannot generalize our findings to the general population of Greece since several regions are missing from the sampling. The food frequency questionnaire was applied once in the selected sample. Another limitation is the misreporting of food items consumed and especially alcohol consumption, due to recall bias or social class of the participants. Moreover, the food frequency questionnaire has been validated in a sample of school teachers, while we have applied it into the general population. This may hide over - or under - estimation of various nutrients estimated.

\section{CONCLUSIONS}

The present work revealed that dietary habits in Greece tend to deviate from the traditional Mediterranean dietary pattern and to resemble those of the westernized countries that are characterized by a high red meat and low fish, vegetable, fruit, dairy and whole grain products consumption. In several countries dietary guidelines have been developed and used as a source of compulsory information not only in public health practice and policy, but in ecology and economy as well (18). The deviation observed in our survey from the National guidelines should carry a clear message to the public health policy makers, in Greece, if we also take into account the protective role of this dietary pattern of human health $(2,6,8,17,19)$. The understanding and the adoption of the recommended consumption of various foods by the people should be a major target of the preventive strategies.

\section{REFERENCES}

1. Nutrition and Food Security Programme 2002-2003. Copenhagen: WHO Regional Office for Europe; 2002.

2. Ministry of Health and Welfare of Greece. Dietary guidelines for adults in Greece. Arch Hellenic Med. 1999;16(5):516-24.

3. Kromhout D, Keys A, Aravanis C, Buzina R, Fidanza F, Giampaoli S, et al. Food consumption patterns in the 1960's in seven countries. Am J Clin Nutr. 1989 May;49(5):889-94.

4. Kafatos A, Kouroumalis I, Vlachonikolis I, Theodorou C, Labadarios D. Coronary heart disease risk factor status of the Cretan urban population in the 1980s. Am J Clin Nutr. 1991;54(3):591-8.

5. DAFNE project [homepage on the Internet]. [cited 2005 Jul 5]. Available from: http://www.nut.uoa.gr/.

6. Trichopoulou A, Costacou T, Bamia C, Trichopoulos D. Adherence to a Mediterranean diet and survival in a Greek population. N Engl J Med. 2003 Jun 26;348(26):2599-608.

7. Pitsavos C, Panagiotakos DB, Chrysohoou C, Stefanadis C. Epidemiology of cardiovascular risk factors in Greece: aims, design and baseline characteristics of the ATTICA study. BMC Public Health. 2003 Oct 20;3:32.

8. Katsouyanni K, Rimm EB, Gnardellis C, Trichopoulos D, Polychronopoulos E, Trichopoulou A. Reproducibility and relative validity of an extensive semi-quantitative food frequency questionnaire using dietary records and biochemical markers among Greek schoolteachers. Int J Epidemiol. 1997;26 Suppl 1:S118-27.

9. Panagiotakos D. Dietary patterns: a Mediterranean diet score and its relation to clinical and biological markers of cardiovascular disease risk. Nutr Metab Cardiov Dis. 2006. (In press.)

10. Trichopoulou A, Georga K. Composition tables of simple and composite foods. Athens: Parisianos Pub; 2003.

11. Moschandreas J, Kafatos A. Food and nutrient intakes of Greek (Cretan) adults. Recent data for food-based dietary guidelines in Greece. Br J Nutr. 1999 Apr;81 Suppl 2:S71-6.

12. CINDI Dietary Guide. EUR/00/5018028.2000. Copenhagen: WHO Regional Office for Europe; 2000.

13. Dietary Guidelines for Americans 2005. 6th ed. Washingthon, DC: U.S. Government Printing Office; 2005.

14. Graca P. Dietary guidelines and food nutrient intakes in Portugal. Br J Nutr. 1999 Apr;81 Suppl 2:S99-103.

15. Volatier JR, Verger P. Recent national French food and nutrient intake data. Br J Nutr. 1999 Apr;81 Suppl 2:S57-9.

16. Scali J, Richard A, Gerber M. Diet profiles in a population sample from Mediterranean southern France. Public Health Nutr. 2001 Apr;4(2):173-82.

17. Knoops KT, de Groot LC, Kromhout D, Perrin AE, Moreiras-Varela O, Menotti A, et al. Mediterranean diet, lifestyle factors, and 10-year mortality in elderly European men and women: the HALE project. JAMA. 2004 Sep 22;292(12):1433-9.

18. Brázdová Z, Ruprich J, Hrubá D, Petráková A. Dietary guidelines in the Czech Republic III.: Challenge for the 3rd millennium. Cent Eur J Publ Health. 2001 Feb;9(1):30-4.

19. Panagiotakos DB, Pitsavos Ch, Chrysohoou Ch, Stefanadis Ch, Toutouzas $\mathrm{P}$. The role of traditional mediterranean type of diet and lifestyle, in the development of acute coronary syndromes: preliminary results from CARDIO 2000 study. Cent Eur J Publ Health. 2002 Jun;10(1-2):11-5.

Received November 22, 2005

Received in revised form and accepted February 20, 2006 